\title{
Morphologic Features of ALK-negative Anaplastic Large Cell Lymphomas With DUSP22 Rearrangements
}

\author{
Rebecca L. King, MD, * Linda N. Dao, MD, ${ }^{*}$ Ellen D. McPhail, MD, * Elaine S. Jaffe, MD, $\dagger$ \\ Jonathan Said, MD, $\ddagger$ Steven H. Swerdlow, MD,§ Christopher A. Sattler, BS,* Rhett P. Ketterling, MD,* \\ Jagmohan S. Sidhu, MD, /| Eric D. Hsi, MD, 9 Shridevi Karikehalli, MD,\# Liuyan Jiang, MD,** \\ Sarah E. Gibson, MD, $\$$ Sarah L. Ondrejka, MD, Alina Nicolae, MD, $\dagger$ William R. Macon, MD,* \\ Surendra Dasari, PhD, $\dagger \dagger$ Edgardo Parrilla Castellar, MD, + t and Andrew L. Feldman, MD*
}

\begin{abstract}
Systemic anaplastic large cell lymphomas (ALCLs) are classified into ALK-positive and ALK-negative types. We recently reported that ALK-negative ALCLs are genetically heterogenous. The largest subset, representing $30 \%$ of cases, had rearrangements of the DUSP22 locus. These cases had favorable outcomes similar to ALK-positive ALCL, and superior to other ALK-negative ALCLs. Here, we examined the morphologic features of these cases in more detail. First, we conducted blinded review of hematoxylin and eosin slides of 108 ALCLs from our previous study, scoring cases for the presence of 3 histologic patterns and 5 cell types. Cases then were unblinded and re-reviewed to understand
\end{abstract}

these features further. DUSP22-rearranged ALCLs were more

From the *Department of Laboratory Medicine and Pathology; $\dagger \dagger$ Division of Biomedical Statistics and Informatics, Mayo Clinic, Rochester, MN; †Hematopathology Section, Laboratory of Pathology, National Cancer Institute, Bethesda, MD; $¥$ Department of Pathology, David Geffen School of Medicine, University of California, Los Angeles, CA; §Division of Hematopathology, University of Pittsburgh Medical Center, Pittsburgh, PA; $\|$ Department of Pathology and Laboratory Medicine, United Health Services Hospitals, Johnson City/ Binghamton; \#Department of Pathology and Laboratory Medicine, Centrex Clinical Laboratories, Utica, NY; Department of Clinical Pathology, Cleveland Clinic, Cleveland, OH; **Department of Laboratory Medicine and Pathology, Mayo Clinic, Jacksonville, FL; and \$DDepartment of Pathology, Duke University, Durham, NC.

Conflicts of Interest and Source of Funding: Supported by Award Numbers R01 CA177734 (A.L.F.) and P50 CA97274 (University of Iowa/Mayo Clinic Lymphoma SPORE) from the National Cancer Institute, by Award Number UL1 TR000135 (Mayo Clinic CTSA) from the National Center for Advancing Translational Science, and by the Predolin Foundation. S.H.S. has accepted a speaker honorarium and meeting travel expenses from Chugai Pharmaceutical Co. E.D.H. has received a speaker honorarium from Seattle Genetics and sponsored research support from Abbvie, Eli Lilly, and Cellerant Therapeutics. The authors have disclosed that they have no significant relationships with, or financial interest in, any commercial companies pertaining to this article.

Correspondence: Andrew L. Feldman, MD, Department of Laboratory Medicine and Pathology, Hilton Building, Room 8-00F, Mayo Clinic, 200 First St. SW, Rochester, MN 55905 (e-mail: feldman. andrew@mayo.edu).

Supplemental Digital Content is available for this article. Direct URL citations appear in the printed text and are provided in the HTML and PDF versions of this article on the journal's Website, www.ajsp.com.

Copyright (C) 2015 Wolters Kluwer Health, Inc. All rights reserved. likely than other ALK-negative ALCLs to have so-called doughnut cells $(23 \%$ vs. $5 \% ; P=0.039)$, less likely to have pleomorphic cells $(23 \%$ vs. $49 \% ; P=0.042)$, and nearly always $(95 \%)$ had areas with sheet-like growth (common pattern). To examine the reproducibility of these findings, we conducted blinded review of hematoxylin and eosin slides of 46 additional ALK-negative ALCLs using a 0 to 3 scoring system to predict likelihood of DUSP22 rearrangement, the results of which correlated strongly with subsequent findings by fluorescence in situ hybridization $(P<0.0001)$. Although all ALCLs share certain morphologic features, ALCLs with DUSP22 rearrangements show significant differences from other ALK-negative ALCLs, typically showing sheets of hallmark cells with doughnut cells and few large pleomorphic cells. These morphologic findings and our previous outcome data suggest that ALK-positive ALCLs and DUSP22-rearranged ALCLs represent prototypical ALCLs, whereas ALCLs lacking rearrangements of both DUSP22 and $A L K$ require further study.

Key Words: anaplastic large cell lymphoma, ALK, DUSP22, TP63

(Am J Surg Pathol 2016;40:36-43)

A naplastic large cell lymphomas (ALCLs) represent a group of CD30-positive T-cell non-Hodgkin lymphomas that vary in their genetics, clinical presentation, and biological behavior. The World Health Organization recognizes 3 distinct types of ALCL: systemic ALK-positive ALCL, systemic ALK-negative ALCL, and primary cutaneous ALCL. ${ }^{1}$ Systemic ALK-positive ALCLs consistently have rearrangements of the $A L K$ gene and have favorable outcomes in most cases after standard combination chemotherapy. ${ }^{2}$ In contrast, systemic ALK-negative ALCLs lack $A L K$ rearrangements and as a whole have inferior outcomes than ALK-positive ALCLs. ${ }^{3} \mathrm{We}$ recently showed, however, that ALK-negative ALCL is a genetically and a clinically heterogenous entity. ${ }^{4}$ Thirty percent of cases bear rearrangements of the DUSP22IRF4 locus on 6p25.3 (DUSP22 rearrangements), ${ }^{5,6}$ and these cases have favorable outcomes similar to those of ALK-positive ALCL. A smaller fraction of patients 
( $\sim 8 \%)$ have rearrangements of $T P 63^{7}$ and have very poor outcomes, whereas the remaining cases lack rearrangements of ALK, DUSP22, and TP63 ("triple-negative" ALCLs) and have intermediate outcomes.

The morphologic features of ALCL have been studied extensively, particularly in ALK-positive cases. Several histologic patterns, including the common, lymphohistiocytic, Hodgkin-like, and small-cell patterns, have been described; the ability to identify $A L K$ rearrangements or expression of the resultant ALK fusion proteins in these cases led to recognition that these patterns could be unified into a single clinicopathologic entity. ${ }^{8}$ Among ALK-negative ALCLs, the lack of a unifying finding such as ALK expression has made the inclusion of variant histologic patterns more challenging, but a spectrum of morphologic features may be seen in this disease, with the exception of a small-cell pattern. ${ }^{9}$ All ALCLs share the feature of containing a cell type with characteristic cytologic features, including a kidney-shaped or horseshoe-shaped nucleus, and designated the hallmark cell. ${ }^{8}$ However, the number of typical hallmark cells varies among cases, as does the number of other cell types, including socalled "doughnut" cells with central nuclear pseudoinclusions, immunoblastic cells, and large, pleomorphic cells that sometimes have nuclei with a "wreath-like" appearance.

Despite the above observations, diagnostic criteria to distinguish ALK-negative ALCL from other CD30-positive T-cell non-Hodgkin lymphomas, particularly peripheral T-cell lymphoma, not otherwise specified (PTCL, NOS), remain imprecise and somewhat controversial. ${ }^{3,9-11}$ Therefore, in our recent study on the genetics of ALK-negative ALCL, ${ }^{4}$ we used a 2 -tiered, blinded review by a panel of expert hematopathologists (E.S.J., J.S., and S.H.S.) to select cases for inclusion. The first tier consisted of assessing whether classic features of ALCL were present on the basis of a hematoxylin and eosin (H\&E)-stained slide and the pattern of CD30 immunostaining. Interestingly, the highest consensus on the presence of classic features of ALCL was observed for cases with DUSP 22 rearrangements. In addition, the highest consensus on a final diagnosis of ALKnegative ALCL was observed for cases with DUSP22 rearrangements after second-tier review, which included additional immunophenotypic data. However, the specific morphologic findings responsible for these associations could not be ascertained because they were not scored prospectively and because the first-tier analysis also included the CD30 staining pattern. Therefore, we undertook the present study to characterize the morphologic features of ALK-negative ALCLs with DUSP22 rearrangements.

\section{METHODS}

Two study groups were analyzed. Study group 1 consisted of 108 systemic ALCLs (77 ALK-negative, 31 ALK-positive) assessed in our previous study. ${ }^{4}$ One ALKpositive ALCL from that study was not available for review. Four ALK-negative ALCLs that had been assessed previously but not reported because of lack of clinical outcome data were included in the present study. Morphologic assessments were performed by 3 hematopathologists
(R.L.K., L.N.D, and E.D.M.) not involved in our previously published series of ALCLs. H\&E slides were reviewed without any additional immunostains. Aside from knowing that all patients carried a diagnosis of ALCL, reviewers were blinded to all additional clinicopathologic data, including ALK status and genetic results. The presence of 3 histologic patterns and 5 cell types were scored independently by each reviewer. The histologic patterns included the common pattern, the lymphohistiocytic pattern, and the Hodgkin-like pattern. ${ }^{8}$ Although the common pattern as used in the World Health Organization classification reflects both the architectural pattern and the cytologic features of the tumor cells, ${ }^{12}$ here we emphasized the characteristic of sheet-like growth as cytologic features were considered separately. Cases with a small-cell pattern were excluded because an ALK-negative form is not recognized. ${ }^{9}$ Cell types assessed were hallmark cells, doughnut cells, immunoblasts, large pleomorphic cells, and wreath-like cells. Each pattern or cell type was scored as "predominant," "present but not predominant," or "absent." The presence of sinusoidal involvement also was noted. Final scores were determined by majority.

After all cases in study group 1 were scored, associations between morphologic findings and genetics were assessed. H\&E sections were re-reviewed jointly by R.L.K., L.N.D, E.D.M., and A.L.F. in the context of the genetic findings to identify a constellation of features most consistently associated with DUSP 22 rearrangements. Reviewers then used the results from both the blinded analysis and the unblinded group review of study group 1 as a knowledge base from which to assess H\&E-stained sections for study group 2, consisting of an independent set of 46 systemic ALK-negative ALCLs of unknown genetic status. Cases were scored on the basis of a histologic score that ranged from 0 to 3 , where 0 was predicted to be least likely to bear a DUSP22 rearrangement and 3 most likely. Discrete criteria for assigning a particular score were not utilized; reviewers used the constellation of features identified from study group 1 to assess the likelihood of DUSP22 rearrangement in a given case. Genetic status was determined as previously described. ${ }^{4}$ All cases were evaluated for DUSP22 rearrangements by fluorescence in situ hybridization (FISH). Cases were also stained for p63, and cases with $>30 \%$ positive tumor cell nuclei were evaluated by both breakapart FISH for TP63 and dual-fusion FISH for TBL1XR1/TP63. Immunostains for p63 and TIA1 were performed as previously described (2/46 cases did not have sections available for TIA1 staining). ${ }^{4}$ Groups were compared using the 2-tailed Fisher exact test, Wilcoxon test, or Cochran-Armitage trend test, as appropriate; $P$ values of $<0.05$ were considered statistically significant. Research was conducted under a protocol approved by the Mayo Clinic Institutional Review Board.

\section{RESULTS}

\section{Study Group 1}

Demographic data for the 108 cases (31 ALK-positive and 77 ALK-negative) in study group 1 are shown in Table 1. Among the ALK-negative ALCLs, there were 
TABLE 1. Demographics and Distribution of Genetic Subtypes in Study Groups 1 and 2

\begin{tabular}{|c|c|c|c|c|c|}
\hline & \multicolumn{5}{|c|}{ ALCL Type } \\
\hline & ALK Positive & $\begin{array}{l}\text { ALK Negative, } \\
\text { All Cases }\end{array}$ & $\begin{array}{c}\text { ALK Negative, } \\
\text { DUSP22 Rearrangement }\end{array}$ & $\begin{array}{c}\text { ALK Negative, } \\
\text { TP63 Rearrangement }\end{array}$ & $\begin{array}{l}\text { ALK Negative, } \\
\text { "Triple Negative" }\end{array}$ \\
\hline \multicolumn{6}{|c|}{ Age, mean (range) (y) } \\
\hline Study group 1 & $32(6-77)$ & $60(24-95)$ & $59(37-77)$ & $52(31-75)$ & $62(24-95)$ \\
\hline Study group 2 & - & $62(32-89)$ & $63(33-81)$ & $70(66-75)$ & $62(32-89)$ \\
\hline \multicolumn{6}{|l|}{ Sex: M:F } \\
\hline Study group 1 & $20: 11$ & $52: 25$ & $15: 7$ & $3: 3$ & $34: 15$ \\
\hline \multicolumn{6}{|c|}{ Distribution of rearrangements: $\mathrm{n}$ ( $\%$ of ALK-negative cases) } \\
\hline Study group 1 & $31(-)^{*}$ & $77(100)$ & $22(29)$ & $6(8)$ & $49(63)$ \\
\hline Study group 2 & - & $46(100)$ & $16(35)$ & $2(4)$ & $28(61)$ \\
\hline
\end{tabular}

*This set of ALK-positive ALCLs was included in study group 1 as a reference, and the number of cases does not reflect the relative frequency of ALK positivity across all ALCLs.

22 with DUSP22 rearrangements $(28 \%), 6$ with TP63 rearrangements $(8 \%)$, and 49 triple-negative ALCLs (64\%). Because TP63 rearrangements were infrequent and were not the subject of this study, these cases and triple-negative cases were combined into a single category of ALK-negative ALCLs lacking DUSP22 rearrangements in subsequent analyses. The common pattern (sheet-like growth) was the most frequently observed in all genetic subtypes (Table 2, Supplemental Table 1, Supplemental Digital Content 1, http://links.lww.com/ PAS/A307). Although not significant, areas with the common pattern were present in DUSP22-rearranged ALCLs more frequently than in other ALK-negative ALCLs $(95 \%$ vs. $80 \% ; P=0.16)$ and at a frequency similar to ALK-positive ALCLs. A representative case of common-pattern ALK-negative ALCL with DUSP22 rearrangement is shown in Figures $1 \mathrm{~A}-\mathrm{D}$. A single case with DUSP22 rearrangement and a lymphohistiocytic pattern was seen (Fig. 1E). DUSP22 rearrangements were not observed in cases with Hodgkin-like features.
The hallmark cell was the predominant cell type in the majority of cases in all subgroups. DUSP22-rearranged ALCLs were more likely than other ALK-negative ALCLs to have doughnut cells $(23 \%$ vs. $5 \% ; P=0.039$; Fig. $1 \mathrm{~A}$, inset) and less likely to have large pleomorphic cells $(23 \%$ vs. $49 \% ; P=0.042$; Figs. $1 \mathrm{~F}, \mathrm{G})$. Large pleomorphic cells were not the predominant cell type in any case with DUSP22 rearrangement. The presence of a sinusoidal growth pattern could not be assessed in a sufficient number of cases to be analyzed further, mostly due to either small size or an extranodal site of the biopsy.

\section{Re-review of Study Group 1}

Upon unblinded joint re-review of the cases in study group 1, the observations noted above were expanded. Cases with DUSP22 rearrangements typically showed sheets of hallmark cells, sometimes including doughnut cells. Hallmark cells can show varying nuclear features, including horseshoe-shaped nuclei with very prominent invaginations and kidney-shaped nuclei with a lesser degree of indentation, which result in a variety of

TABLE 2. Morphologic Findings in ALCLs, Study Group 1

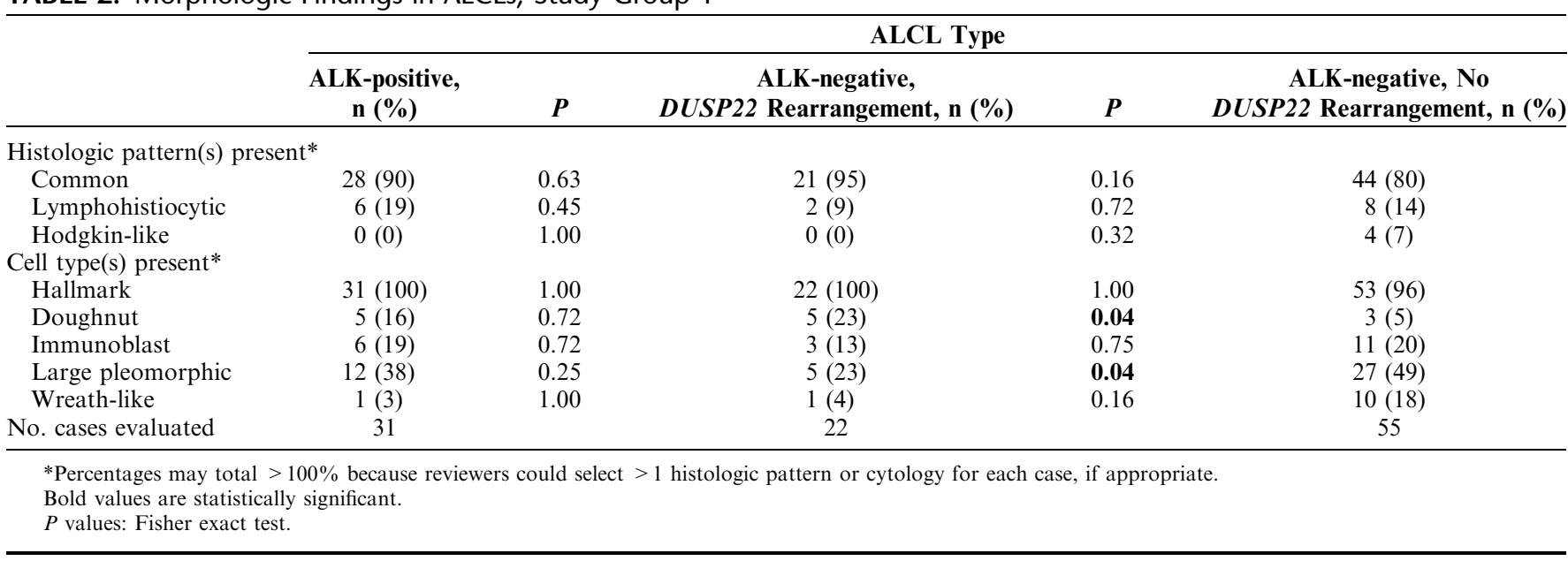



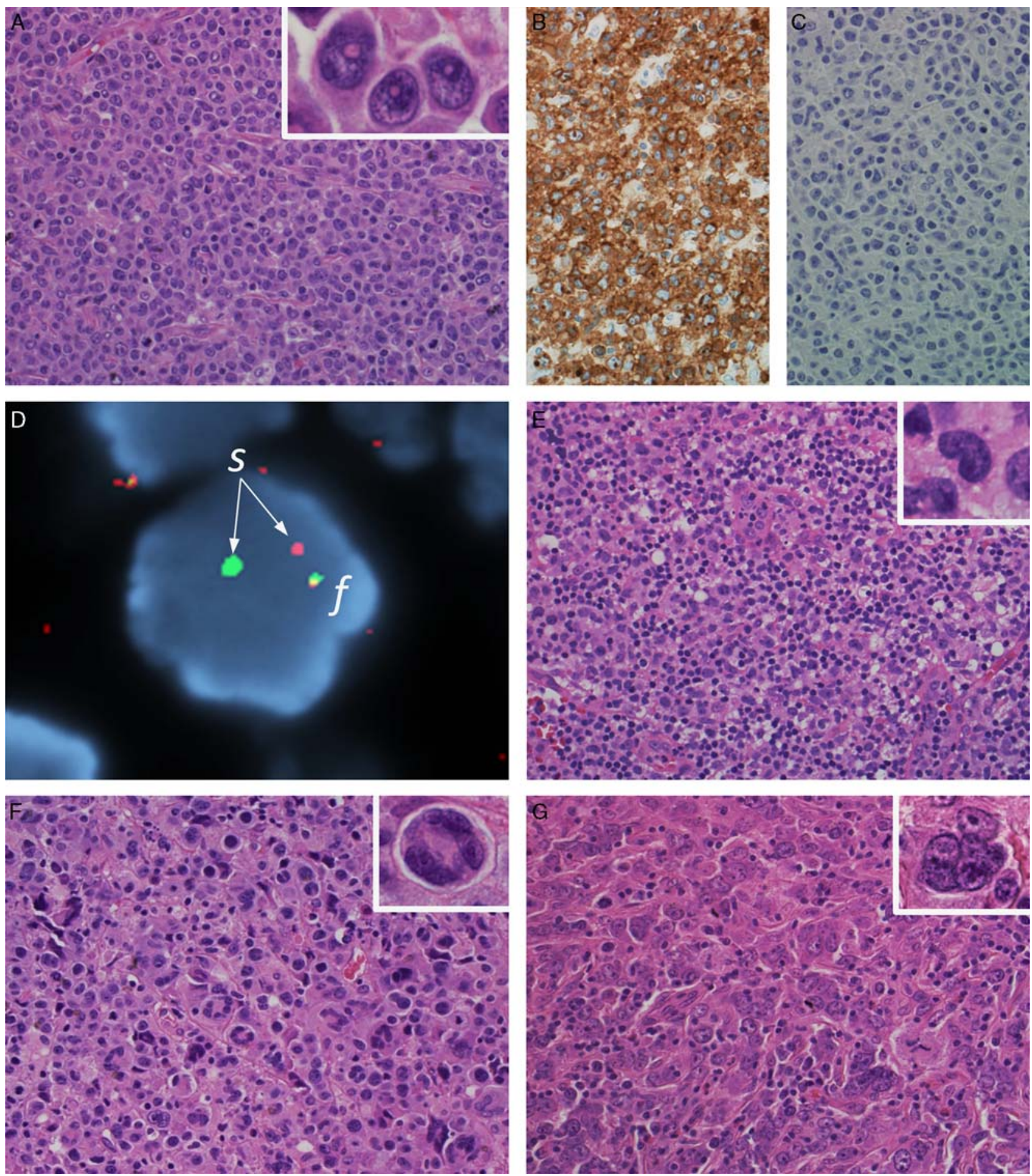

FIGURE 1. Morphologic patterns in ALK-negative ALCL. A, Typical case of ALK-negative ALCL with DUSP22 rearrangement (H\&E). This case represents the so-called "common" pattern, with sheets of tumor cells including numerous hallmark cells (inset, left; H\&E) and occasional "doughnut" cells (inset, right). The tumor cells are strongly and uniformly positive for CD30 (B) and negative for ALK (C). D, FISH using a breakapart probe to the DUSP22 locus shows a rearrangement, with 1 normal fusion signal $(f)$ and an abnormal split signal (s). E, Sole example from study group 1 of ALK-negative ALCL with DUSP22 rearrangement and a lymphohistiocytic pattern (H\&E). The tumor cells are scattered in a background rich in small lymphocytes and histiocytes. Hallmark cells are present (inset; H\&E). F, ALK-negative ALCL with large pleomorphic cells (H\&E), including cells with wreath-like nuclei (inset; $H \& E)$. This case was a "triple-negative" ALCL. G, Another triple-negative ALCL composed predominantly of large pleomorphic cells (H\&E). Cells with wreath-like nuclei were infrequent in this case, but other multinucleate forms were easily identified (inset; H\&E). Large pleomorphic cells could be seen occasionally in DUSP22-rearranged ALCLs, but were not the predominant cell type. 

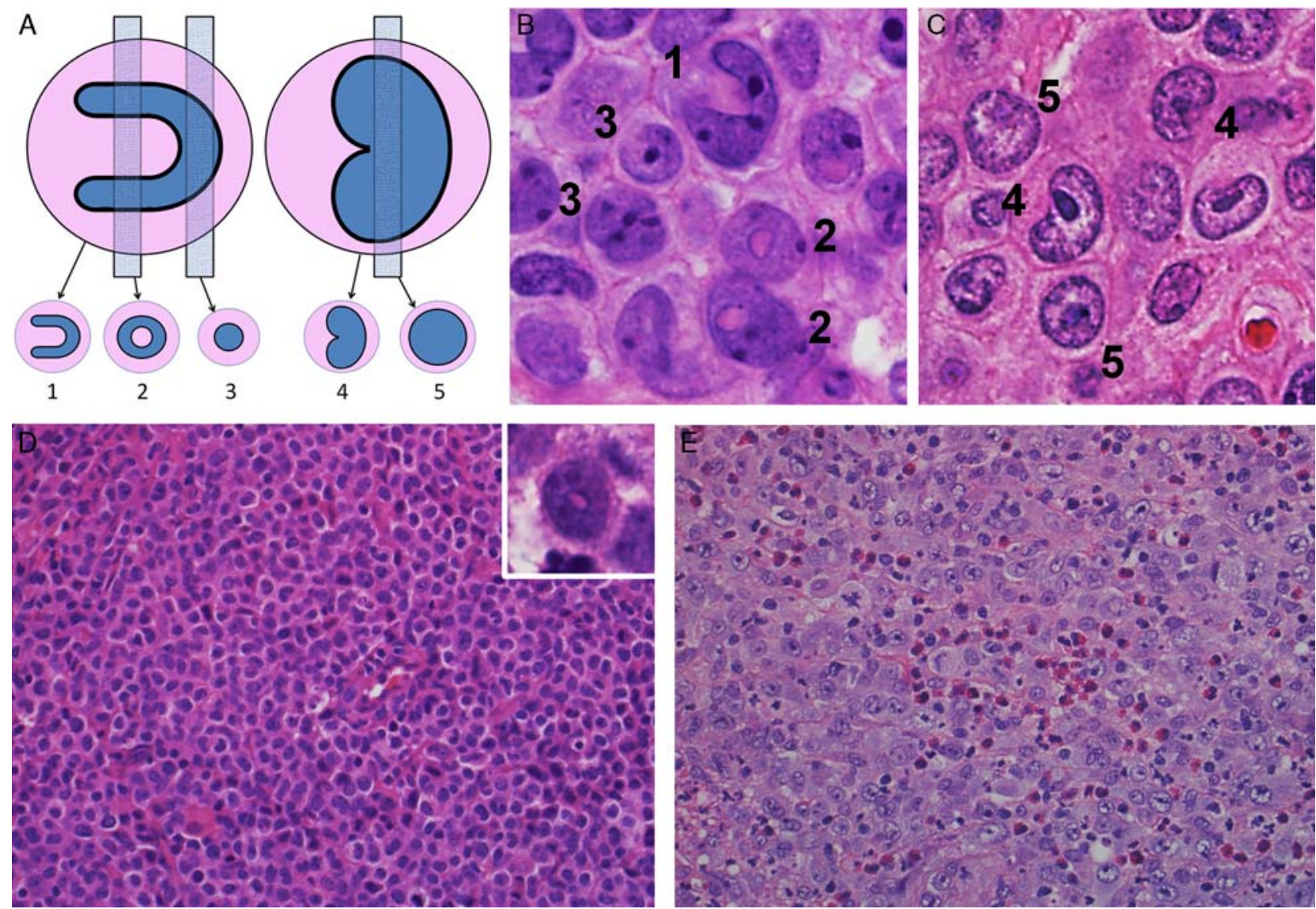

FIGURE 2. Additional morphologic features in ALK-negative ALCL. A, Hallmark cells show a spectrum of features; nuclei with very prominent invaginations are horseshoe-shaped (left), whereas a lesser degree of indentation results in a kidney-shaped nucleus (right). These give rise to different appearances depending on the plane of section. B, ALCLs with DUSP22 rearrangements were noted to have prominent nuclear invagination, sometimes giving rise to numerous doughnut cells, as in this case (H\&E). Numerals denote cells with cytologic features as shown in (A). C, This case of triple-negative showed a common pattern with predominantly hallmark cells, which had kidney-shaped rather than horseshoe-shaped nuclei (H\&E). D, Some DUSP22-rearranged ALCLs showed hallmark cells with relatively small, somewhat hyperchromatic nuclei in sheets without admixed acute inflammatory cells (H\&E). A finding very suggestive of the presence of a DUSP22 rearrangement was the combination of this smaller cell size with the appearance of doughnut cells ("mini-doughnuts"; inset; H\&E). E, A finding uncommon in DUSP22-rearranged ALCLs was the admixture of granulocytes or eosinophils, as in this triple-negative ALCL (H\&E). Also note the larger cell size and more open nuclear chromatin compared with (D).

appearances depending upon the plane of sectioning through a given cell (Fig. 2A). Therefore, the 3-dimensional configuration of the cells must be reconstructed mentally on the basis of evaluation of the full spectrum of nuclear features observed. Consistent with the increased presence of doughnut cells in DUSP22-rearranged ALCLs, hallmark cells in these cases tended to show more nuclear invagination than in other ALK-negative ALCLs, which tended to be more kidney-shaped (Figs. 2B, C). Some accentuation of nuclear indentation could be seen even in DUSP22-rearranged ALCLs without doughnut cells. It also was noted upon re-review that the tumor cells in some cases with DUSP22 rearrangements appeared slightly smaller than the tumor cells of other ALCLs of the common pattern, and the nuclear chromatin was slightly more condensed (Fig. 2D). The cell size often was quite uniform, and admixed inflammatory cells were absent, giving these cases a monotonous appearance. Although the cells were small to medium in size, the features were not those of the small-cell variant of ALK-positive ALCL, in which only rare hallmark cells can be identified and the majority of cells have irregular nuclear contours and moderate clear to weakly basophilic cytoplasm. ${ }^{13}$ In contrast, these cases of DUSP22-rearranged ALCLs had a relatively monomorphic population of typical hallmark cells with smaller cell size. The combination of this smaller cell size with the appearance of doughnut cells (mini-doughnuts) was very suggestive of the presence of a DUSP22 rearrangement.

The presence of large pleomorphic cells was considered atypical of cases with DUSP22 rearrangements. This included not only the wreath-like nuclei that were 

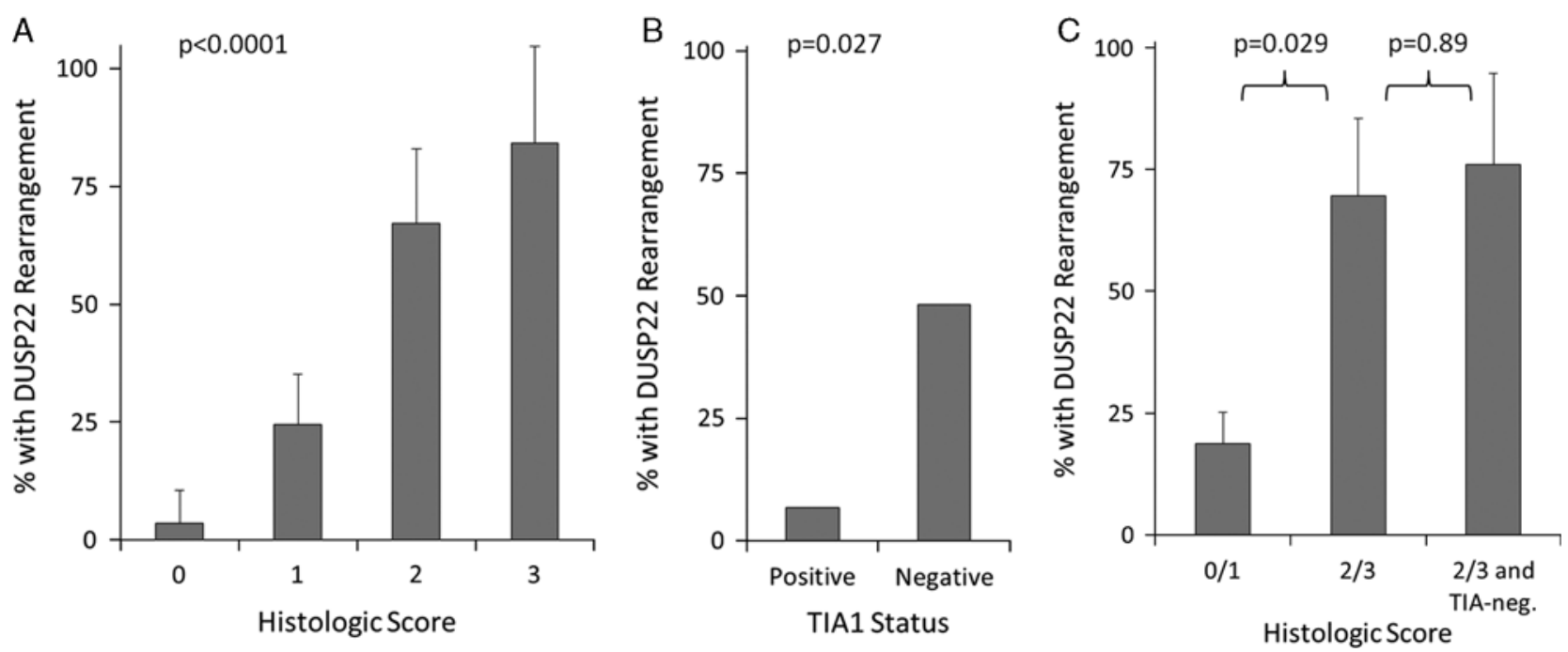

FIGURE 3. Correlates of a histologic score in study group $2(n=46)$. A, Histologic score correlated closely with the frequency of DUSP22 rearrangement in ALK-negative ALCLs. B, TIA1 expression also was inversely associated with the presence of DUSP22 rearrangement. C, A histologic score of 2 or 3 was associated with the presence of DUSP22 rearrangements, but adding TIA1 negativity to the histologic score did not increase the rearrangement frequency significantly.

scored prospectively but a wide range of cytologic features corresponding to bizarre nuclear shapes, multinucleation, and Reed-Sternberg-like features. In general, fewer background inflammatory cells were seen in DUSP22-rearranged cases, and specifically the presence of eosinophils was uncommon (Fig. 2E).

\section{Study Group 2}

Of the 46 ALK-negative ALCLs in study group 2, there were 16 with DUSP22 rearrangements (35\%), 2 with TP63 rearrangements $(4 \%)$, and 28 triple-negative ALCLs $(61 \%)$. This distribution was similar to the relative frequencies of these genetic subtypes in our previous report, ${ }^{4}$ as reflected by study group 1 (Table 1). Using the constellation of histologic features identified from blinded and unblinded review of study group 1, reviewers provided histologic scores that correlated significantly with the presence of DUSP22 rearrangements. Specifically, the mean frequencies $( \pm \mathrm{SD})$ of DUSP22 rearrangements in cases given scores of $0,1,2$, and 3 were $3.5 \% \pm 7.0 \%$, $24.5 \% \pm 10.7 \%, 67.3 \% \pm 15.7 \%$, and $84.3 \% \pm 20.5 \%$, respectively $(P<0.0001$; Fig. 3A). Therefore, despite the subjective nature of this scoring system, reviewers could identify and validate the constellation of histologic features associated with DUSP22 rearrangements in an independent group of ALK-negative ALCLs.

We previously showed that, unlike many ALCLs, those with DUSP22 rearrangements typically lack cytotoxic marker expression. ${ }^{4,5}$ The strongest association was observed for TIA1 expression, assessed by immunohistochemistry. ${ }^{4}$ Therefore, we sought to determine whether TIA1 expression could add to the ability of the histologic score to predict the presence of a DUSP22 rearrangement. The frequencies of DUSP22 rearrangements in ALK-negative ALCLs that were positive and negative for TIA1 were
$6.7 \%$ and $48.3 \%$, respectively $(P=0.027$; Fig. 3B), further validating our previous findings. A histologic score of 2 or 3 was associated with a $69.5 \% \pm 16.0 \%$ frequency of DUSP22 rearrangement, compared with $18.8 \% \pm 6.3 \%$ for a histologic score of 0 or $1(P=0.029$; Fig. 3C). No significant increase in the predictive ability of a histologic score of 2 or 3 was observed when negativity for TIA1 was included as an additional criterion $(76.0 \% \pm 18.6 \%$; $P=0.89$ ).

\section{DISCUSSION}

This study is the first to dissect the morphologic features of systemic ALK-negative ALCLs with DUSP22 rearrangements. Next to $A L K$ rearrangements, DUSP 22 rearrangements are the second most common chromosomal rearrangements in ALCL. To date, DUSP22 rearrangements have been mutually exclusive with $A L K$ rearrangements, and both genetic subsets show similarly favorable outcomes compared with other systemic ALCLs and PTCLs; specifically, 5-year overall survival rates were $90 \%$ for DUSP22-rearranged ALCLs and $85 \%$ for ALK-positive ALCLs, compared with $17 \%$ and $42 \%$ for TP63-rearranged ALCLs and triple-negative ALCLs, respectively. ${ }^{4}$ Here, we have demonstrated that DUSP22rearranged ALCLs have a unique and reproducible constellation of morphologic features recognizable on $\mathrm{H} \& \mathrm{E}$ stains, further supporting the concept that these cases represent a clinicopathologically distinct subset of ALCLs.

The histologic features identified as associated with DUSP22 rearrangements in this study recapitulate the classic features of ALCL described in earlier morphologic descriptions of this entity. ${ }^{8,14-16}$ Particularly, these cases typically showed sheet-like growth of hallmark cells with 
a tendency to have prominent nuclear indentations, yielding a nuclear contour that is more horseshoe-shaped than kidney-shaped. This prominent degree of indentation accounts for the appearance of doughnut cells, which are sectioned perpendicular to the axis of indentation. ${ }^{17}$ Because most ALCL cells are relatively large compared with the standard thickness of H\&E sections, the frequency of doughnut cells is proportional to the extent of nuclear indentation. Doughnut cells were seen with similar frequency in DUSP22-rearranged ALCLs and ALK-positive ALCLs, but were uncommon in the other genetic subgroups. Notably absent in ALCLs with DUSP22 rearrangements were the large pleomorphic cells in their various manifestations (wreath-like, Reed-Sternberg-like, etc.) that have been reported in both ALK-positive and ALK-negative ALCLs. Furthermore, DUSP22-rearranged cases with a lymphohistiocytic pattern were infrequent and no cases with a Hodgkin-like pattern were seen. Although cases with a Hodgkin-like pattern were included in the original cohort, more recent studies suggest that such cases be excluded from ALK-negative ALCL. ${ }^{10,11}$ Further studies, including gene expression profiling and mutational analysis, will be necessary to resolve the best way to classify these atypical cases. Taken together, our findings suggest that ALK-negative ALCLs with DUSP22 rearrangements represent prototypical ALCLs with a morphologic spectrum narrower than that of ALK-positive ALCLs.

The presence of $A L K$ rearrangements, determined by immunohistochemistry or genetic tests, has allowed inclusion of cases with variant histologic patterns in the category of ALK-positive ALCL. ${ }^{8,12}$ The question arises whether detection of DUSP22 rearrangements could play a similar role in the diagnosis of ALK-negative ALCL. As discussed above, the definition of ALK-negative ALCL is evolving, and a certain degree of subjectivity remains in the distinction from other mature T-cell lymphomas expressing CD $30 .{ }^{9}$ Although we have observed DUSP22 rearrangements in cases with a submitting diagnosis of CD30-positive PTCL, NOS, ${ }^{5}$ all such cases were reclassified as ALK-negative ALCL upon independent, blinded review in our subsequent study. ${ }^{4}$ Therefore, identifying DUSP 22 rearrangements by FISH might - in the proper clinicopathologic setting - be a useful tool in the diagnosis of ALK-negative ALCL, defining a group of cases with characteristic morphology, immunophenotype, and clinical behavior. ${ }^{4}$ Of note, DUSP 22 rearrangements can also be seen in $28 \%$ of primary cutaneous ALCLs and even rarely in lymphomatoid papulosis. ${ }^{18,19}$ Primary cutaneous ALCLs also occasionally may be ALK-positive, albeit less commonly. ${ }^{20}$ Therefore, interpretation of any ALCL with skin involvement requires correlation with staging and other clinical data.

Although this study focused primarily on DUSP22rearranged ALCLs, a few comments on the remaining subsets of ALK-negative ALCLs are warranted. First, TP63-rearranged ALCLs are rare, and experience is limited. As in the case of DUSP22 rearrangements, cases with TP63 rearrangements originally diagnosed as PTCL, NOS were reclassified as ALK-negative ALCLs during central review in our previous study. ${ }^{4}$ However, this reclassification was made with a slightly lower degree of consensus than for DUSP22-rearranged cases, and in our opinion too few TP63-rearranged ALCLs have been reported to draw conclusions about their general characteristics. Nevertheless, like DUSP22-rearranged cases, TP63rearranged ALCLs did not tend to have large pleomorphic cells, which were observed almost exclusively in triplenegative ALCLs and ALK-positive ALCLs. Not all triplenegative ALCLs had these features, suggesting that further heterogeneity may exist within this subgroup independent of the rearrangements studied here.

\section{REFERENCES}

1. Swerdlow S, Campo E, Harris N, et al. WHO Classification of Tumours of Haematopoietic and Lymphoid Tissues. Lyon: International Agency for Research on Cancer; 2008.

2. Gascoyne RD, Aoun $\mathrm{P}, \mathrm{Wu} \mathrm{D}$, et al. Prognostic significance of anaplastic lymphoma kinase (ALK) protein expression in adults with anaplastic large cell lymphoma. Blood. 1999;93: 3913-3921.

3. Savage KJ, Harris NL, Vose JM, et al. ALK - anaplastic large-cell lymphoma is clinically and immunophenotypically different from both ALK + ALCL and peripheral T-cell lymphoma, not otherwise specified: report from the International Peripheral T-Cell Lymphoma Project. Blood. 2008;111:5496-5504.

4. Parrilla Castellar ER, Jaffe ES, Said JW, et al. ALK-negative anaplastic large cell lymphoma is a genetically heterogeneous disease with widely disparate clinical outcomes. Blood. 2014;124:1473-1480.

5. Feldman AL, Law M, Remstein ED, et al. Recurrent translocations involving the IRF4 oncogene locus in peripheral T-cell lymphomas. Leukemia. 2009;23:574-580.

6. Feldman AL, Dogan A, Smith DI, et al. Discovery of recurrent $\mathrm{t}(6 ; 7)(\mathrm{p} 25.3 ; \mathrm{q} 32.3)$ translocations in ALK-negative anaplastic large cell lymphomas by massively-parallel genomic sequencing. Blood. 2011;117:915-919.

7. Vasmatzis G, Johnson SH, Knudson RA, et al. Genome-wide analysis reveals recurrent structural abnormalities of TP63 and other p53-related genes in peripheral T-cell lymphomas. Blood. 2012;120: 2280-2289.

8. Benharroch D, Meguerian-Bedoyan Z, Lamant L, et al. ALKpositive lymphoma: a single disease with a broad spectrum of morphology. Blood. 1998;91:2076-2084.

9. Mason DY, Harris NL, Delsol G, et al. Anaplastic large cell lymphoma, ALK-negative. In: Swerdlow S, Campo E, Harris N, et al, eds. WHO Classification of Tumours of Haematopoietic and Lymphoid Tissues. Lyon: International Agency for Research on Cancer; 2008:317-319.

10. Klapper W. What is a true ALCL? Blood. 2014;124:1385-1386.

11. Attygalle AD, Cabecadas J, Gaulard P, et al. Peripheral T-cell and NK-cell lymphomas and their mimics; taking a step forwardreport on the lymphoma workshop of the XVIth meeting of the European Association for Haematopathology and the Society for Hematopathology. Histopathology. 2014;64:171-199.

12. Delsol G, Falini B, Muller-Hermelink HK, et al. Anaplastic large cell lymphoma, ALK-positive. In: Swerdlow S, Campo E, Harris N, et al, eds. WHO Classification of Tumours of Haematopoietic and Lymphoid Tissues. Lyon: International Agency for Research on Cancer; 2008:312-316.

13. Kinney M, Collins R, Greer J, et al. A small-cell-predominant variant of primary Ki-1 (CD30) + T-cell lymphoma. Am J Surg Pathol. 1993;17:859-868.

14. Stein H, Mason D, Gerdes J, et al. The expression of the Hodgkin's disease associated antigen $\mathrm{Ki}-1$ in reactive and neoplastic lymphoid tissue: evidence that Reed-Sternberg cells and histiocytic malignancies are derived from activated lymphoid cells. Blood. 1985;66: 848-858. 
15. Agnarsson B, Kadin M. Ki-1 positive large cell lymphoma: a morphologic and immunologic study of 19 cases. Am J Surg Pathol. 1988;12:264-274.

16. Bitter MA, Franklin WA, Larson RA, et al. Morphology in Ki1(CD30)-positive non-Hodgkin's lymphoma is correlated with clinical features and the presence of a unique chromosomal abnormality, t(2;5)(p23;q35). Am J Surg Pathol. 1990;14:305-316.

17. Jaffe ES. Post-thymic T-cell lymphomas. In: Jaffe ES, ed. Surgical Pathology of the Lymph Nodes and Related Organs. Philadelphia, PA: W.B. Saunders; 1995:344-389.
18. Wada DA, Law ME, Hsi ED, et al. Specificity of IRF4 translocations for primary cutaneous anaplastic large cell lymphoma: a multicenter study of 204 skin biopsies. Mod Pathol. 2011;24:596-605.

19. Karai LJ, Kadin ME, Hsi ED, et al. Chromosomal rearrangements of 6p25.3 define a new subtype of lymphomatoid papulosis. Am J Surg Pathol. 2013;37:1173-1181.

20. Oschlies I, Lisfeld J, Lamant L, et al. ALK-positive anaplastic large cell lymphoma limited to the skin: clinical, histopathological and molecular analysis of 6 pediatric cases. A report from the ALCL99 study. Haematologica. 2013;98:50-56. 